\title{
EVOLUCIÓN DEL FÓSFORO DISPONIBLE A DISTINTOS NIVELES DE COMPACTACIÓN POR TRÁFICO AGRÍCOLA EN UN ARGIUDOL TÍPICO
}

\section{EVOLUTION OF AVAILABLE PHOSPHORUS AT DIFFERENT LEVELS OF COMPACTION BY AGRICULTURAL TRAFFIC IN A TYPICAL ARGIUDOL}

\author{
Juan Martín Gueçaimburu' ${ }^{1 *}$, Juan Manuel Vázquez ${ }^{1}$, Facundo Tancredi², Gisela Paola Reposo ${ }^{1}$, \\ Verónica Rojo ${ }^{1}$, Maximiliano Martínez ${ }^{1}$, y Rafael Mario Introcaso ${ }^{1}$ \\ ${ }^{1}$ Departamento de Tecnología, Universidad Nacional de Luján, C.P. 6700, Luján, Argentina \\ ${ }^{2}$ Universidad Nacional de Luján, C.P. 6700, Luján, Argentina \\ * Autor para correspondencia E-mail: jmguecaimburu@yahoo.com.ar
}

\section{RESUMEN}

El nivel de fósforo (P) disponible en el suelo es una variable dinámica, influenciada por las propiedades del suelo, la planta y las condiciones ambientales. Los procesos de degradación física afectan la estructura del suelo y la disponibilidad de los nutrientes. Este trabajo tuvo como objetivo evaluar la dinámica del $\mathrm{P}$ en el tiempo, a diferentes niveles de compactación de suelo, inducidos por tráfico agrícola. Los tratamientos fueron: T0: testigo (sin pasadas de tractor); T1 (6 pasadas) y T2 (12 pasadas). Se muestreó a 0-10 $\mathrm{cm}$ de profundidad: $P$, densidad aparente (DAP), materia orgánica (MO) y $\mathrm{pH}$, con intervalos de 60 días, hasta los 180 días. Los análisis de $\mathrm{MO}$ y $\mathrm{pH}$, se realizaron de manera complementaria, ya que son factores estrechamente relacionados con la dinámica del P. La DAP aumentó en relación a la intensidad del tráfico agrícola, y la disponibilidad de fosforo presentó una relación inversa con la DAP en los muestreos realizados a los 60 y 120 días, periodo en el que se registraron elevadas precipitaciones. A los 180 días ya no se registraron diferencias en el $P$ disponible entre tratamientos de compactación. El análisis de correlación del $\mathrm{pH}$ y la materia orgánica con el $\mathrm{P}$ disponible no fue significativo en las condiciones del ensayo.

Palabras clave: densidad aparente, tráfico agrícola, fertilidad, fósforo disponible.

\section{ABSTRACT}

The level of phosphorus (P) available in the soil is a dynamic variable, influenced by soil properties, plant and environmental conditions. Physical degradation processes affect soil structure and nutrient availability. The aim of this study was to evaluate the dynamics of $P$ over time, at different levels of soil compaction by agricultural traffic. The following treatments were used: T0: control (without passes of tractor); T1 (6 passes) and T2 (12 passes). Soil samples were taken from 0-10 cm depth at intervals of 60 days up to 180 days. $P$, bulk density (DAP), organic matter (MO) and $\mathrm{pH}$ were evaluated. $\mathrm{MO}$ and $\mathrm{pH}$ analyzes were performed in a complementary manner as they are factors closely related to $\mathbf{P}$ dynamics. DAP increased with agricultural traffic intensity, while phosphorus availability showed an inverse relationship with DAP in the samples taken at 60 and 120 days; high rainfall was recorded during this period. After 180 days, no differences were found in available $\mathrm{P}$ between compaction treatments. The correlation analysis of $\mathrm{pH}$ and organic matter with available $P$ was not significant for the test conditions.

Key words: bulk density, agricultural traffic, fertility.

Recibido: 05 septiembre 2018. Aceptado: 12 noviembre 2018. 


\section{INTRODUCCIÓN}

La región pampeana argentina se caracteriza por poseer contenidos variables de $\mathrm{P}$ total (fósforo total) en función de su material original. Sin embargo, la intensificación agrícola, el aumento considerable de los rendimientos y el bajo empleo de fertilizantes, han contribuido a generar deficiencias del elemento en zonas que tradicionalmente estaban bien provistas de este nutriente (Viglizo et al, 2011). Las fuentes de P y su distribución son críticas para la sostenibilidad de las prácticas agrícolas, ya que se requiere para favorecer la formación de semillas, el desarrollo radicular, la fuerza de las pajas en los cereales y la maduración de los cultivos (Rincón y Gutiérrez, 2012)

Desde el punto de vista químico el $\mathrm{P}$ puede encontrarse como P inorgánico y como P orgánico. El $\mathrm{P}$ inorgánico se clasifica de acuerdo a su disponibilidad para las plantas en: i) $\mathrm{P}$ disponible, que es la forma aprovechable en forma inmediata, es decir son fosfatos en la solución del suelo; ii) en $\mathrm{P}$ intercambiable, también llamado lábil o adsorbido, cuya disponibilidad es más lenta que el anterior; y iii) por último el $\mathrm{P}$ insoluble, que está formando parte de los minerales primarios y secundarios, y constituye la gran reserva de $\mathrm{P}$ inorgánico en el suelo (Johnston y Syers, 2009). La insolubilización del $\mathrm{P}$ se puede deber a la precipitación como fosfatos cálcicos en medios alcalinos, o como fosfatos de hierro y aluminio en medios ácidos; en cuanto al $\mathrm{P}$ orgánico, su principal fuente es la constituida por los residuos vegetales y animales que se adicionan al suelo (Sanzano 2006). Los compuestos fosfatados más importantes de la materia orgánica $(\mathrm{MO})$ son las nucleoproteínas, los fosfolípidos y los fosfoazúcares. La mineralización de la MO es lenta y por vía microbiana, requiriendo condiciones óptimas de temperatura, aproximada a $25-30^{\circ} \mathrm{C}$, un $\mathrm{pH}$ neutro y una humedad cercana a capacidad de campo. Este P no está disponible para las plantas en forma inmediata, lo que se conoce como P lábil (Suñer y Galantini, 2013).

El P, después del nitrógeno (N), es el nutriente que más frecuentemente limita la producción de cultivos. Es considerado esencial para el crecimiento y desarrollo de las plantas. Las plantas toman el P de la solución del suelo como fosfato monoácido $\left(\mathrm{PO}_{4} \mathrm{H}_{2}^{-}\right)$y como fosfato diácido $\left(\mathrm{PO}_{4} \mathrm{H}^{=}\right)$; el primero en mayor proporción que el segundo. Normalmente, esta solución es demasiado pobre para sostener un cultivo, y debe ser realimentada continuamente desde las formas más insolubles de $\mathrm{P}$ a medida que los cultivos lo extraen de la solución de suelo (Syers et al., 2008). En condiciones neutras a alcalinas los minerales secundarios principalmente formados son fosfatos de calcio. En condiciones ácidas, el $\mathrm{P}$ precipita con hierro y aluminio. Con el aumento del $\mathrm{pH}$ los fosfatos de aluminio y hierro aumentan su solubilidad, mientras que los de calcio disminuyen, alcanzando la mayor concentración de $\mathrm{P}$ en la solución del suelo a $\mathrm{pH}$ entre 6-6.5 (Arizmendi-Galicia et al, 2011).

En cuanto a $\mathrm{P}$ disponible, Campitelli et al (2010), indicaron que el contenido de P disponible en el suelo es una variable dinámica, fuertemente influenciada por las propiedades del suelo, la planta y las condiciones ambientales. Cualquier cambio en las propiedades del suelo se encuentra relacionado con la concentración de $\mathrm{P}$ en solución.

Como se expresó previamente, y por lo establecido por García et al. (2009), desde un punto de vista agronómico, las plantas absorben el $\mathrm{P}$ desde la fracción soluble en el suelo, la que a su vez es reabastecida por una fracción lábil de naturaleza orgánica e inorgánica. Esta fracción de $\mathrm{P}$ lábil está en equilibrio con fracciones moderadamente lábiles y de muy baja labilidad. Dada la baja concentración de $\mathrm{P}$ soluble, el abastecimiento de $\mathrm{P}$ desde la fracción lábil es de extrema importancia para garantizar un adecuado suministro de $\mathrm{P}$ a las plantas (Syers et al. 2008).

El diagnóstico de la fertilidad física es complementario al diagnóstico de la fertilidad química, y su diagnóstico es un paso previo que debe tomarse antes de diagnosticar la disponibilidad de nutrientes, y eventualmente así poder recomendar prácticas correctivas, como la fertilización (Taboada y Álvarez, 2008).

El mantenimiento de la estructura del suelo garantiza una adecuada calidad física del mismo. No obstante, dado que las propiedades físicasedáficas son el resultado del equilibrio entre las tres fases que componen el suelo (sólido, agua y aire), los indicadores físicos de calidad de suelo suelen incluir determinaciones que relacionan a éstas fases entre sí, como es el caso de la densidad aparente (DAP) o la retención de humedad entre los puntos de capacidad de campo y de marchitez permanente (Taboada y Álvarez, 2008). Si bien en la actualidad existen mejores indicadores físicos que la DAP, este es de los pocos parámetros que pueden determinar los productores agropecuarios a campo.

Independientemente de cuál sea el proceso de degradación física, su resultado es la disminución dela disponibilidad de los nutrientes. Los procesos de degradación del suelo, inciden directamente en la disponibilidad del P. La compactación puede afectar los procesos químicos de liberación de nutrientes, y procesos físicos como la infiltración y circulación del agua (Campitelli et al, 2010). 
Keller y Håkansson (2010), definieron a la DAP como la masa de suelo seco por unidad de volumen $\left(\mathrm{kg} \mathrm{m}^{-3}\right)$. Este sería uno de los parámetros que describe la compactación del suelo, representando la relación entre sólidos y espacio poroso. Reyes (2007) considera que un suelo está compactado cuando su macroporosidad es tan baja que restringe la aireación. Si se incrementa la DAP, la resistencia mecánica tiende a aumentar y la porosidad del suelo tiende a disminuir con lo cual se reduce la conductividad de aire y de agua. El método comúnmente utilizado para la determinación de DAP y que es altamente confiable, es el conocido como "método del cilindro", que consiste en introducir un cilindro metálico en el suelo y luego de enrasarlo una vez extraído, determinar la masa de suelo seco que quedó en su interior (Agostini et al., 2014).

Como se ha dicho, la compactación aumenta la resistencia mecánica del suelo, disminuyendo el volumen que puede ser explorado por las raíces $\mathrm{y}$, por lo tanto, disminuye el acceso espacial a nutrientes y agua. Este proceso es más importante para nutrientes como el $\mathrm{P}$, que tiene poca movilidad en el suelo. El transporte de los nutrientes desde el suelo a las raíces se realiza por dos mecanismos principales: flujo masal y difusión. Sin embargo, en el caso de los nutrientes poco móviles, sólo pequeñas cantidades llegan a las raíces por flujo de masa. Así, más del 95\% del $\mathrm{P}$ absorbido llega a las raíces por difusión. La compactación altera el tamaño y distribución del sistema poroso, afectando el contenido volumétrico de agua del suelo y así el movimiento de $\mathrm{P}$ en el suelo, disminuyendo las fracciones más lábiles de $\mathrm{P}$ (Cabeza, 2011).

La compactación también limita el P disponible por decrecimiento del espesor de las láminas de agua entre las partículas de suelo, entre las cuales el P soluble se mueve hacia la superficie radicular (Cahueñas y Cesar 2015).

Reintam et al. (2005), encontraron que plantas de cebada fueron altamente afectadas por el déficit de nutrientes en suelos compactados, dependiendo del nivel de DAP, lo que avala el estudio de este factor sobre la oferta de nutrientes en el suelo. Forsythe et al. (2005), obtuvieron en sus estudios que la absorción de nutrientes, especialmente de N, P y K en maíz (Zea mays L.) se redujeron claramente con el aumento de la compactación.

El pH de los suelos afecta la reactividad de los componentes del suelo con el ortofosfato, y establece la presencia y abundancia relativa de los cationes que pueden precipitar con el P (Sims y Pierzynski, 2005).

Los mayores niveles de compactación del suelo, tanto superficiales como subsuperficiales, generados por el tránsito de equipos agrícolas, pisoteo del ganado, encostramientos, agregado a factores intrínsecos de los propios suelos, aumentan su degradación física. Esta degradación puede ser atenuada mediante prácticas de manejo, como por ejemplo, el tránsito controlado de maquinaria, la labranza reducida, la labranza vertical, pastoreos rotativos, etc.

La escases de prácticas conservacionistas pueden generar una merma en la fertilidad del suelo y como consecuencia de ello una baja en los rendimientos de los cultivos. Este trabajo tuvo por objetivo estudiar la relación entre la disponibilidad del fosforo y la compactación inducida en el suelo luego del tránsito de máquinas agrícolas.

\section{MATERIALES Y MÉTODOS}

Localización. El trabajo se realizó en el Campo Experimental de la Universidad Nacional de

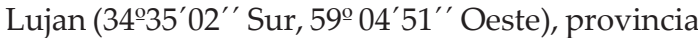
de Buenos Aires, Argentina.

Caracterización inicial. El suelo donde se llevó a cabo el trabajo es un Argiudol típico con capacidad de uso IIw (Soil Survey Staff, 1999), de textura franco-limosa en superficie, bien provisto de $\mathrm{MO}(3,00 \%)$, con $\mathrm{pH}$ moderadamente ácido (pH: 5,60$)$ y disponibilidad de $\mathrm{P}$ asimilable por Kurtz y Bray (1945) de 9,14 mg kg-1. El sitio del ensayo fue roturado con una rastra de discos en los días previos a la realización del ensayo.

Tratamientos. Se realizaron 3 tratamientos esperando obtener 3 estados diferentes de compactación: T0 (testigo) el sector que no se sometió a ningún tipo de compactación inducida; T1 tratamiento de 6 pasadas de tractor sobre una misma huella; y T2 en que se realizó un tratamiento de 12 pasadas. Estas son las cantidades mínimas y máximas que frecuentan en un ciclo de cultivo dado por tránsito de rodados para la siembra, fertilización, pulverizaciones, cosecha, etc., como lo expresa Balbuena et al. (2003). Todas las pasadas se efectuaron en el mismo día para garantizar uniformidad en las condiciones sobre las variables cuantificadas, sobre todo para tener mínimas variaciones en la capacidad portante del suelo y su consiguiente efecto sobre la compactación del mismo.

Evaluaciones. Las determinaciones fueron realizadas sobre suelo arado sin transitar para el T0, y sobre la huella del tractor para los T1 y T2. Los métodos utilizados fueron los siguientes: DAP, por Método del Cilindro (Blake y Hartge, 1986); P disponible, por método de Kurtz \& Bray I 
(Kurtz y Bray, 1945); MO por método de Walkley \& Black (Walkley y Black, 1934); y pH (Black et al, 1965).

Al inicio del ensayo, definido como día 0 , se realizaron 3 determinaciones de DAP en cada parcela de cada tratamiento, para corroborar si se encontraban diferencias sobre la compactación en el suelo. De cada parcela de cada tratamiento se tomó una muestra de suelo de 0 a $10 \mathrm{~cm}$ de profundidad, compuesta de 10 submuestras, en las que se determinó $\mathrm{P}$ disponible, $\mathrm{MO}$ y $\mathrm{pH}$, siendo estos últimos complementarios dada la estrecha relación entre estos parámetros y la dinámica del $\mathrm{P}$ en el suelo.

Luego se repitieron las mediciones de DAP y $P$ disponible para cada parcela a los 60, 120 y 180 días.

La parcela se mantuvo con el pasto corto con desmalezado manual y pulverización con mochila durante el ensayo.

Características del tractor. Se utilizó un tractor de tracción delantera asistida (FWA) de cuatro ruedas motrices, de $103 \mathrm{CV}$ en el motor, un peso total de $4890 \mathrm{~kg}$. Más detalles en Tabla 1.

Diseño experimental. El diseño experimental fue en parcelas divididas, realizando tres repeticiones de los distintos tratamientos. Las parcelas experimentales tuvieron una longitud de 40 metros y un ancho de 15 metros, dentro de las cuales se establecieron las parcelas de medición en el sector medio de las mismas. Los resultados fueron analizados mediante ANOVA aplicando el test de Tukey $(\mathrm{p}<0,05)$.

\section{RESULTADOS Y DISCUSIÓN}

Densidad aparente. El rango de valores de DAP observados se presentan en la Tabla 2 y son comunes a situaciones de Argiudoles típicos compactados y laboreados, como los encontrados por Mur y Balbuena (2014) en suelos de similares características. Se promediaron las repeticiones de cada tratamiento para obtener un valor medio de cada muestreo y cada tratamiento. Las diferencias que se encontraron entre los tratamientos fueron estadísticamente significativas $(p<0,05)$ siendo bien marcadas en el primer y tercer muestreo, y con ciertas variaciones en el segundo y cuarto (Tabla 2).

En cuanto al análisis entre muestreos, no se observan diferencias significativas entre las distintas fechas para los tratamientos de 6 y 12 pasadas del tractor, lo que permite inferir que para esos niveles de compactación el suelo no tiene capacidad de revertir el incremento de la densidad aparente en los primeros meses (Tabla 2). En el caso del T0 se ve un aumento de la DAP en el segundo muestreo, el cual puede haberse producido por el reordenamiento $\mathrm{y}$ reempaquetamiento de las partículas de suelo disqueado y una posterior expansión atribuibles a factores como la contracción y expansión del suelo por la variación en los contenidos de humedad (Tabla 2) (Hossne y Américo, 2008).

Fósforo disponible. Al inicio del ensayo la disponibilidad de $\mathrm{P}$ que presentaba el total de las parcelas sin disturbios era de 9,14 mg $\mathrm{kg}^{-1}$. Se promediaron los valores de $\mathrm{P}$ disponible

Tabla 1. Características del tractor usado en el experimento de efecto de la compactación en la disponibilidad de fósforo.

Table 1. Characteristics of the tractor used in the experiment about the effect of compaction on the availability of phosphorus.

\begin{tabular}{lc}
\hline Parámetros y Unidades & Tractor Pesado \\
\hline Diseño tractivo & FWA \\
Potencia en el motor $(\mathrm{kW})$ & 76,8 \\
Peso total $(\mathrm{kg})$ & 4891 \\
Peso eje delantero $(\mathrm{kg})$ & 1935 \\
Peso eje trasero $(\mathrm{kg})$ & 2955 \\
Neumáticos delanteros & $14,9-24$ \\
Neumáticos traseros & $18,4-34$ \\
Área de contacto de neumático trasera $\left(\mathrm{m}^{2}\right)$ & 0,168 \\
Presión en el área de contacto rueda trasera/suelo $(\mathrm{kPa})$ & 86,25 \\
\hline
\end{tabular}


Tabla 2. Valores de DAP $\left(\mathrm{kg} \mathrm{cm}^{-3}\right)$ entre los tratamientos de tráfico y los muestreos. Table 2 . Values of bulk density (DAP) $\left(\mathrm{kg} \mathrm{cm}^{-3}\right)$ in the treatments and sampling.

\begin{tabular}{ccccc}
\hline Tratamiento & $\mathbf{1}^{\mathbf{0}}$ muestreo Día $\mathbf{0}$ & $\mathbf{2}^{\mathbf{0}}$ muestreo Día $\mathbf{6 0}$ & $\mathbf{3}^{\mathbf{0}}$ muestreo Día $\mathbf{1 2 0}$ & $\mathbf{4}^{\mathbf{o}}$ muestreo Día 180 \\
\hline $\mathrm{T} 0$ & $1,14 \mathrm{aA}$ & $1,24 \mathrm{aB}$ & $1,14 \mathrm{aA}$ & $1,16 \mathrm{aA}$ \\
$\mathrm{T} 1$ & $1,30 \mathrm{bA}$ & $1,27 \mathrm{aA}$ & $1,25 \mathrm{bA}$ & $1,31 \mathrm{bA}$ \\
$\mathrm{T} 2$ & $1,37 \mathrm{cA}$ & $1,36 \mathrm{bA}$ & $1,33 \mathrm{cA}$ & $1,36 \mathrm{bA}$ \\
\hline
\end{tabular}

Letras minúsculas distintas en sentido vertical indican diferencias significativas entre tratamientos $(\mathrm{P}<0,05$ Tukey) y letras mayúsculas distintas en sentido horizontal indican diferencias significativos entre fechas de muestreo ( $P$ $<0,05$ Tukey).

obtenidos en las 3 repeticiones de cada muestreo. Los muestreos 2 y 3 presentan una reducción del $\mathrm{P}$ disponible con el incremento del grado de compactación (Figs. 1 y 2), siendo significativas las diferencias entre los tratamientos de $6 \mathrm{y}$ 12 pasadas en el segundo muestreo y entre los tres tratamientos para el tercer muestreo. En el cuarto muestreo luego de 180 días de realizados los tratamientos de compactación, ya no se observan diferencias significativas (Tabla 3).

De acuerdo a los resultados entre muestreos dentro de cada tratamiento, se esperaba una disminución de la disponibilidad de $\mathrm{P}$ en el período de tiempo estudiado, debido al efecto negativo de la compactación en la tasa de difusión de oxígeno y movilidad del agua (Arvidsson y Håkansson, 2014), siendo mayor la disponibilidad al día 0 y menor al día 180 . Se observó dicha tendencia negativa durante el período bajo estudio pero todos los tratamientos tuvieron un aumento en la disponibilidad del $P$ en el tercer muestreo al día 120. Se esperaba que la disponibilidad del $\mathrm{P}$ fuera inversamente proporcional al aumento de la DAP y al tiempo, siendo el T0 el de mayor disponibilidad, ya que su menor DAP proporcionaría, en principio, un mejor sistema poroso (reflejado en los valores obtenidos en la DAP) que brindaría una mayor disponibilidad de iones fosfatos a la solución de suelo, según lo expone Syers et al. (2008). En función de lo expresado y bajo el mismo criterio en T1 se esperaba que hubiese menor cantidad de $\mathrm{P}$ disponible que en $\mathrm{T} 0$, y por último que en $\mathrm{T} 2$ hubiese menos $\mathrm{P}$ disponible que los demás tratamientos. Coincidiendo con lo expresado por Snyder y Slaton (2002), se cree que el aumento en la disponibilidad de $\mathrm{P}$ a los 120 días fue provocado por el exceso hídrico proporcionado por las lluvias de enero y febrero de 2014, con precipitaciones acumuladas de 568 $\mathrm{mm}$, siendo el período previo al tercer muestreo. Snyder y Slaton (2002), sostienen que en el suelo en condiciones de saturación hídrica aumenta el $\mathrm{P}$ disponible, fundamentalmente porque los

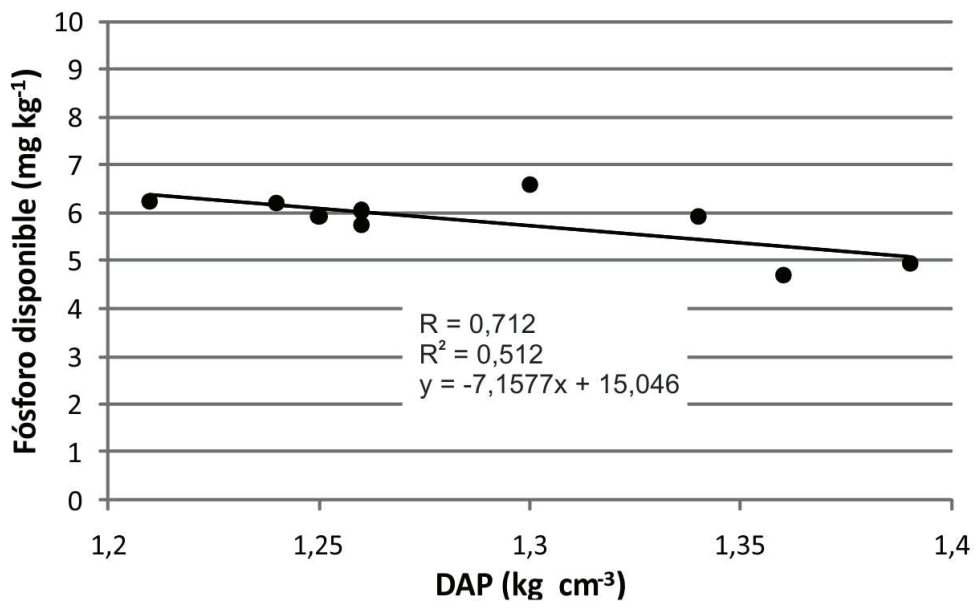

Significativo al nivel 0,05.

Fig. 1. Relación entre la DAP y el P disponible en el segundo muestreo para las diferentes intensidades de compactación.

Fig. 1. Relation between DAP and available $P$ at 2 nd sampling for different soil compaction intensities. 


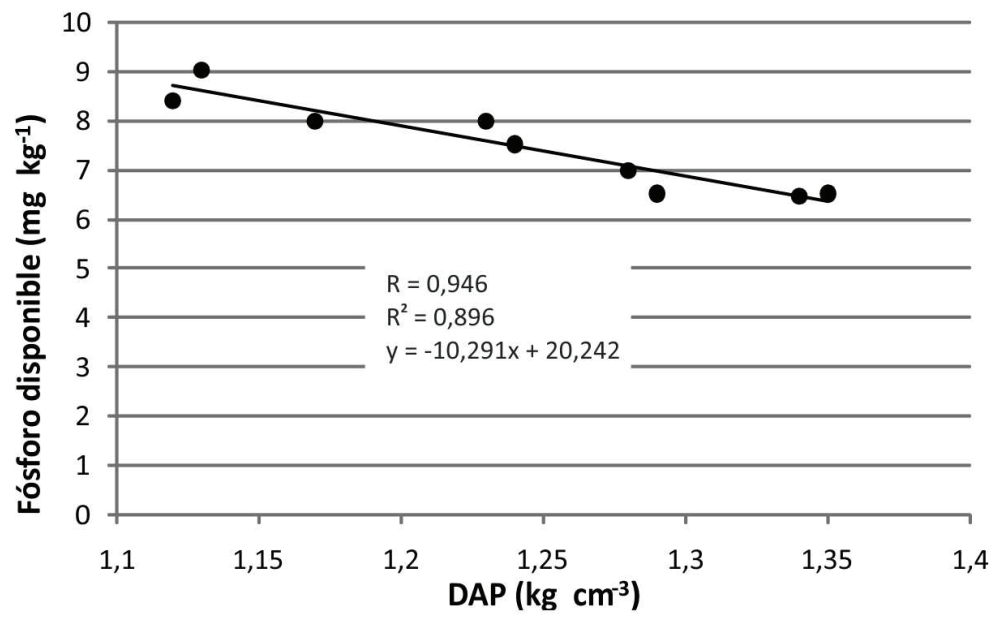

Significativo al nivel 0,01.

Fig. 2. Relación entre la DAP y el P disponible en el tercer muestreo para las diferentes intensidades de compactación.

Fig. 2. Relation between DAP and available $P$ at 3 rd sampling for different soil compaction intensities.

Tabla 3. Valores de $\mathrm{P}$ disponible ( $\left.\mathrm{mg} \mathrm{kg}^{-1}\right)$ en los tratamientos y fechas de muestreo.

Table 3. Values of available $P\left(\mathrm{mg} \mathrm{kg}^{-1}\right)$ in treatmentss and sampling.

\begin{tabular}{cccc}
\hline Tratamiento & $\mathbf{2}^{\mathbf{0}}$ muestreo Día $\mathbf{6 0}$ & $\mathbf{3}^{\mathbf{o}}$ muestreo Día $\mathbf{1 2 0}$ & $\mathbf{4}^{\mathbf{o}}$ muestreo Día $\mathbf{1 8 0}$ \\
\hline T0 & $6,03 \mathrm{abA}$ & $8,47 \mathrm{aB}$ & $3,07 \mathrm{aC}$ \\
T1 & $6,17 \mathrm{aA}$ & $7,50 \mathrm{bA}$ & $5,00 \mathrm{aA}$ \\
T2 & $5,17 \mathrm{bAB}$ & $6,50 \mathrm{cB}$ & $3,67 \mathrm{aA}$ \\
\hline
\end{tabular}

Letras minúsculas distintas en sentido vertical indican diferencias significativas entre tratamientos $(\mathrm{P}<0,05$ Tukey) y letras mayúsculas distintas en sentido horizontal indican diferencias significativos entre fechas de muestreo $(\mathrm{P}<0,05$ Tukey).

fosfatos férricos se reducen a fosfatos ferrosos y que esta disponibilidad se reduce luego del drenaje del suelo. En este sentido, en el último muestreo se obtuvieron los menores valores de $\mathrm{P}$ disponible para todos los tratamientos. Es posible que la reducción del $\mathrm{P}$ disponible al aumentar los valores de DAP en el tercer muestreo, haya sido mediada por condiciones más reductoras en las parcelas más compactadas.

La disponibilidad del P disminuyó en todos los tratamientos durante el período establecido pero sin poderle atribuir con mayor grado de certeza dicho efecto al aumento del nivel de compactación inducida.

Relación entre la densidad aparente y el fósforo disponible. Se observó una alta concordancia entre las diferencias significativas de los valores de DAP y las diferencias significativas de los valores de $\mathrm{P}$ disponible de esas mismas muestras (Tablas 2 y 3 ). Esto hizo creer que existió la dinámica planteada entre estos dos factores. Según lo expresado anteriormente, se estudiaron las variables mediante análisis de correlación lineal para ver si existía covariación conjunta de ambos factores. Las Figs. 1, 2 y 3, ilustran la relación entre la DAP y el $\mathrm{P}$ disponible en los muestreos $2^{\circ}, 3^{\circ}$ y $4^{\circ}$, respectivamente (cada figura incluye las medias de las parcelas de los tres tratamientos). En los muestreos 2 y 3 (Figs. 1 y 2 ) se observa claramente una reducción de la disponibilidad del fósforo al aumentar la DAP, mientras que en el último muestreo la relación no es significativa.

Materia orgánica. La condición previa de la parcela era de 3,00\% de $\mathrm{MO}$ al inicio del ensayo. El análisis de la dinámica de los valores de MO no estaba dentro de los objetivos, pero se quiso observar que estos se hallaran en un rango que no incidiera negativamente en la disponibilidad del P. Los valores máximos y mínimos oscilaron 


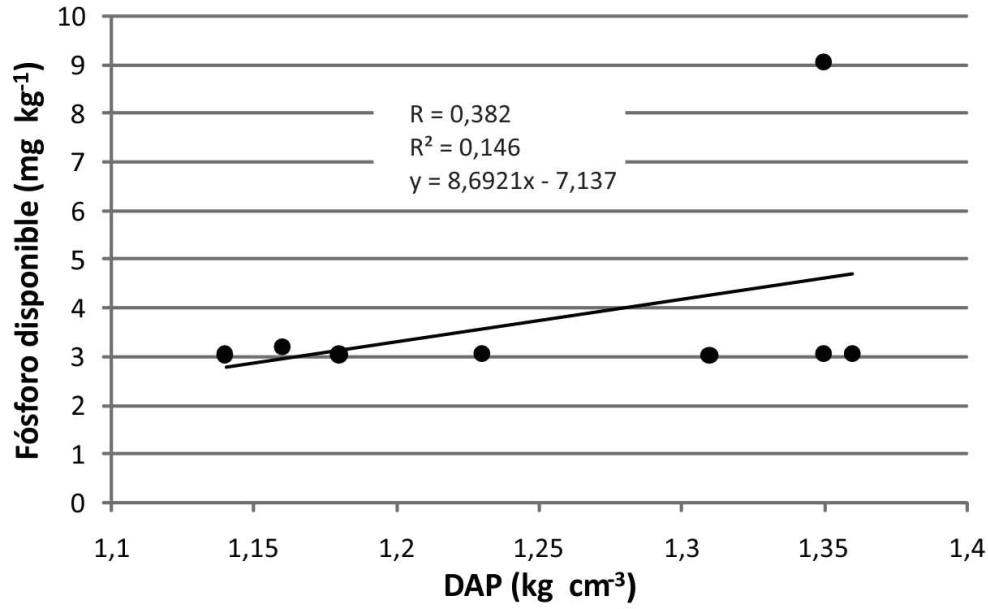

No significativo al nivel 0,5.

Fig. 3. Relación entre la DAP y el P disponible en el cuarto muestreo para las diferentes intensidades de compactación.

Fig. 3. Relation between DAP and available $P$ at 4 th sampling for different soil compaction intensities.

entre $3,00 \%$ y 3,90\%. No se encontraron diferencias significativas entre los tratamientos. El análisis de correlación entre la materia orgánica y el $\mathrm{P}$ disponible no fue significativo al nivel de 0,05 .

pH. Al día 0 la parcela presentaba un $\mathrm{pH}$ de 5,60. Los valores sucesivos se hallaron entre mínimos de 5,00 y máximos de 6,00. En este caso tampoco se quiso estudiar su comportamiento en el tiempo sino que se quería tener como información auxiliar, por la posible incidencia sobre la disponibilidad del $\mathrm{P}$, ya que es uno de los factores que interfiere en la disponibilidad de este nutriente (Sharpley, 2000). El análisis de correlación entre el $\mathrm{pH}$ y el $\mathrm{P}$ disponible no fue significativo al nivel de 0,05.

\section{CONCLUSIONES}

Los valores de DAP obtenidos mostraron aumento a medida que se elevaban la cantidad de pasadas de tractor.

La DAP se mantuvo estable en el tiempo dentro de cada tratamiento, salvo el T0 que tuvo cierta dinámica debida a factores intrínsecos y extrínsecos que actuaron sobre el suelo suelto. Hubo disminuciones de $\mathrm{P}$ respecto a la situación inicial de las parcelas, constatando una reducción en el $\mathrm{P}$ disponible en los primeros centímetros de suelo. Se encontró a los 60 y 120 días que los dos tratamientos con menor DAP tuvieron mayor disponibilidad de $\mathrm{P}$.

El último muestreo fue el que mostró menor disponibilidad de $\mathrm{P}$ en los tres tratamientos, lo que indica un agotamiento del P en solución, pero no mostró diferencias entre suelos con diferentes niveles de compactación inducida.

Más allá del grado de compactación, la disponibilidad del $\mathrm{P}$ fue baja para todos los tratamientos y no hubo diferencias significativas entre ellos.

$\mathrm{Al}$ estudiar las variables DAP y $\mathrm{P}$ disponible en conjunto, se halló, mediante los análisis de correlación, una dependencia aceptable para el segundo y tercer muestreo, con una clara relación inversamente proporcional entre las variables, pero una correlación débil para el cuarto.

\section{LITERATURA CITADA}

Agostini, M.D.L.Á., M.G. Monterubbianesi, G.A. Studdert, and S. Maurette. 2014. Un método simple y práctico para la determinación de densidad aparente. Ciencia del Suelo 32(2):171-176.

Arizmendi-Galicia, N., P. Rivera-Ortiz, F.D.L. Cruz-Salazar, B.I. Castro-Meza, y F. de la Garza-Requena. 2011. Lixiviación de hierro quelatado en suelos calcáreos. Terra Latinoamericana 29(3):231-237.

Arvidsson, J., and I. Håkansson, 2014. Response of different crops to soil compaction. Shortterm effects in Swedish field experiments. Soil and Tillage Research 138:56-63.

Black, C.A., D.D. Evans, and R.C. Dinauer. 1965. Methods of soil analysis. Vol. 9. p. 653-708. American Society of Agronomy, Madison, Wisconsin, USA. 
Blake, G.R., and K.H. Hartge. 1986. Bulk density. p. 363-375. In A. Klute (ed.) Methods of soil analysis. Part $1.2^{\text {nd }}$. ed. Soil Science Society of America, Madison, Wisconsin, USA.

Balbuena, R., G. Botta, L. Draghi, H. Rosatto, y C. Dagostino. 2003. Compactación de suelos. Efectos del tránsito del tractor en sistemas de siembra directa. Spanish Journal of Agricultural Research 1(2):75-80.

Cabeza, R. 2011. Degradación del suelo y sus efectos en la dinámica del fósforo. $174 \mathrm{p}$. Simposio Nacional de la Ciencia del Suelo. Sociedad Chilena de la Ciencia del Suelo, Boletín № 24. p. 107-112

Centeno Cahueñas, J.C. 2015. Influencia de la profundidad del fertirriego por goteo, sobre el sistema radicular y el rendimiento de dos poblaciones de tomate (Lycopersicum esculentum Mil). cv. Mariana. Tabacundo, Pichincha. 154 p. Tesis de Ingeniero Agrónomo. Universidad Central del Ecuador, Faculad de Agronomía,Quito, Ecuador. .

Campitelli, P., A. Aoki, O. Gudelj, A. Rubenacker, y R. Sereno. 2010. Selección de indicadores de calidad de suelo para determinar los efectos del uso y prácticas agrícolas en un área piloto de la región central de Córdoba. Ciencia del Suelo 28(2):223-231.

Díaz-Zorita, M., y M. Basanta. 1999. Efectos de seis años de labranzas en un Hapludol del noroeste de Buenos Aires, Argentina. Ciencia del Suelo 17(1):31-36.

Forsythe, W., F. Sancho, y M. Villatoro. 2005. Efecto de la compactación de suelos sobre el rendimiento del maíz en tres localidades de Costa Rica. Agronomía Costarricense: Revista de Ciencias Agrícolas 29(3):175-185.

García, F.O., I.A. Ciampitti, G. Rubio, y L.I. Picone. 2009. Fertilización fosfatada en Argentina. Actualidad, manejo y perspectivas. p. 1011. Actas CD Jornadas nacionales "Sistemas productivos sustentables: $\mathrm{P}, \mathrm{N}, \mathrm{y}$ cultivos de cobertura". Bahia Blanca, Buenos Aires, Argentina.

Hamza, M.A. and W.K. Anderson. 2005. Soil compaction in cropping systems: a review of the nature, causes and possible solutions. Soil and Tillage Research 82(2):121-145.

Hillel, D. 1998. Environmental soil physics: Fundamentals, applications, and environmental considerations. 771 p. Academic Press, San Diego, California, USA.

Hossne, G., y J. Américo. 2008. La densidad aparente y sus implicaciones agrícolas en el proceso expansión/contracción del suelo. Terra Latinoamericana 26(3):195-202.
Johnston, A.E., and J.K. Syers. 2009. A new approach to assessing phosphorus use efficiency in agriculture. Better Crops 93(3):14-15.

Keller, T., and I. Håkansson. 2010. Estimation of reference bulk density from soil particle size distribution and soil organic matter content. Geoderma 154:398-406.

Kurtz, L.T., and R.H. Bray. 1945. Determination of total, organic, and available forms of phosphorus in soils. Soil Science 59(1):39-46.

Mur, M., y R.H. Balbuena. 2014. Compactación de un suelo argiudol típico por tráfico en un sistema de producción de forrajes. Ciencia del Suelo 32(1):1-12.

Reintam, E., J. Kuht, H. Loogus, E. Nugis, and K. Trükmann. 2005. Soil compaction and fertilisation effects on nutrient content and cellular fluid $\mathrm{pH}$ of spring barley (Hordeum vulgare L.). Agronomy Research 3(2):189202.

Reyes, W. 2007. Evaluación de condiciones y riesgos de compactación en cuatro series de suelo bajo uso agrícola en Venezuela. Crozatía 8(1):63-72.

Richmond, P., y S. Rillo. 2006. Evaluación del efecto de la compactación por el rodado de maquinarias sobre algunas propiedades físicas del suelo y el cultivo de trigo en siembra directa. INPOFOS Informaciones Agronómicas 32:22-25.

Rincón, L.E.C., y F.A.A. Gutiérrez. 2012. Dinámica del ciclo del nitrógeno y fósforo en suelos. Revista Colombiana de Biotecnología 14(1):285-295.

Sanzano, A. 2006. El fósforo del suelo. Química del suelo. Disponible en https://www. edafologia.org/app/download/7956239776/ El+Fosforo+del+suelo.pdf?t=1494945225. (Consulta 5 de marzo 2019).

Sharpley A. 2000. Phosphorous availability. p. D18-D38. In M.E. Sumner (ed.) Handbook of Soil Science. CRC, Boca Raton, Florida, USA.

Sims, J., and G. Pierzynski. 2005. Chemistry of phosphorus in soils. p. 151-192. In M. Tabatabay and D. Sparks (eds.) Chemical Processes in Soils. Book Series 8. Soil Science Society of America, Madison, Wisconsin, USA.

Snyder C.S., and N.A. Slaton. 2002. Effects of soil flooding and drying on phosphorous reactions. In News and views newsletter. Potash \& Phosphate Institute and the Potash \& Potash Institute of Canada. Avalilable at http:// www.ipni.net/ppiweb/ppinews.nsf/0/BFC425FCAE6ACE4E85256B90005BD114/\$FILE/ Flooded\%20Soils.pdf (Accessed 15 March 2018). 
Soil Survey Staff. 1999. Soil taxonomy: A basic system of soil classification for making and interpreting soil surveys. $2^{\text {nd }}$. ed. Natural Resources Conservation Service, Handbook 436. U.S. Department of Agriculture, Washington DC, USA.

Suñer, L., y J. Galantini. 2013. Importancia del equilibrio de las formas de fósforo edáfico. p. 67-73. En J. Galantini (ed.) Ciencia y experiencia para una siembra directa sustentable en los ambientes frágiles del S y SO Bonaerense. AAPRESID, Rosario, Argentina.

Syers, J.K., A.E. Johnston, and D. Curtin. 2008. Efficiency of soil and fertilizer phosphorus use. FAO Fertilizer and Plant Nutrition Bulletin 18. FAO, United Nations, Rome, Italy.

Taboada, M., y C. Álvarez. 2008. Fertilidad física de los suelos. Ed. Facultad Agronomía (UBA). Buenos Aires, Argentina.
Viglizzo, E.F., F.C. Frank, L.V. Carreno, E.G. Jobbagy, H. Pereyra, J. Clatt, and M.F. Ricard. 2011. Ecological and environmental footprint of 50 years of agricultural expansion in Argentina. Global Change Biology 17(2):959973.

Walkley, A., and I.A. Black. 1934. An examination of the Degtjareff method for determining soil organic matter, and a proposed modification of the chromic acid titration method. Soil Science 37(1):29-38. 